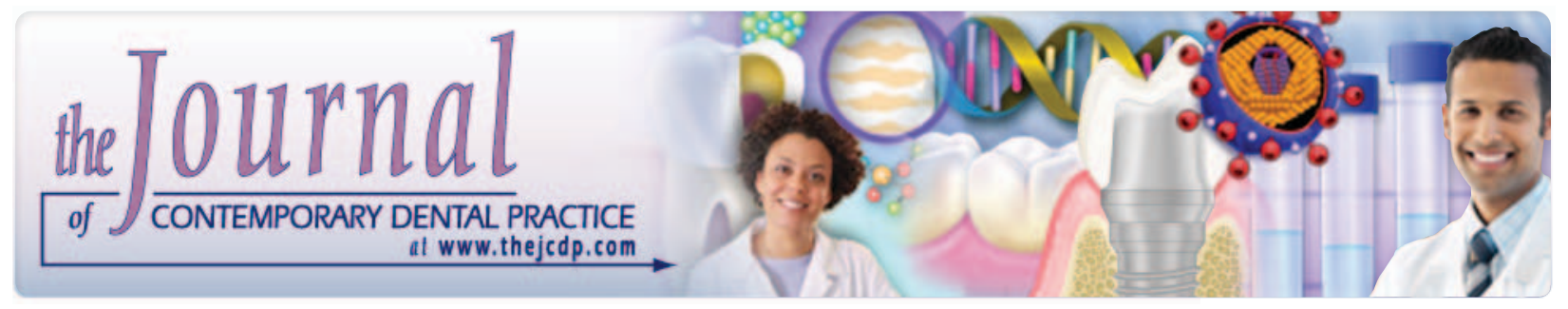

\title{
Dental Treatment Duration as an Indicator of the Behavior of 3- to 9- Year-old Pediatric Patients in Clinical Dental Settings
}

Naser AsI Aminabadi, DDS, MSc; Sina Ghertasi Oskouei, DDS; Ramin Mostofi Zadeh Farahani, DDS

\begin{abstract}
Aim: The aim of this study was to evaluate the age-specific effect of treatment duration on pediatric patient behavior.

Methods and Materials: A total of 450 children 3 to 9 years of age were allocated into six consecutive age groups $(n=75$ for each group). All children received dental treatment procedures which included the fabrication of a Type 1 composite resin restoration, then a dental prophylaxis followed by fluoride therapy with each procedure requiring an average of 20 minutes. The children's behavior was evaluated at the end of each treatment period using the sound, eye, and motor (SEM) scale.
\end{abstract}

Results: The results of a mixed analysis of variance (ANOVA) indicated a significant main effect of treatment duration, $F(1.69,255.01)=$ 188.29, $\mathrm{P}<0.001$, and of chronological age, $F(1,144)=115.82, P<0.001$. Moreover, the main effects of time and chronological age was qualified by a significant interaction between time and chronological age, $F(8.86,255.01)=$ $115.82, \mathrm{P}<0.001$. The beta weights $(0.64$ for age versus 0.44 for time) suggest chronological age contributes the most to predicting the behavior of children during dental treatment followed by the duration of treatment.

Conclusion: Treatment duration may affect the behavior of pediatric patients parallel with chronological age and, thus, should be considered in the arrangement of the treatment plan.

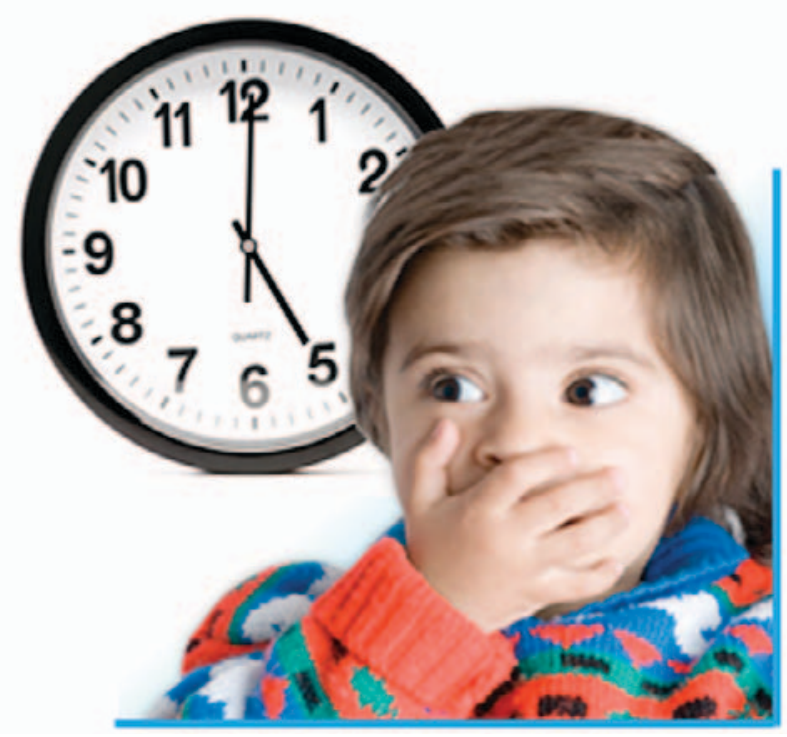

Clinical Significance: The findings of this study suggest appropriate pediatric behavior management should include thoughtful scheduling of appointments according to a treatment plan formulated with consideration of the effects of age and appointment length.

Keywords: Behavior, chronological age, pediatric, treatment plan

Citation: Aminabadi NA, Oskouei SG, Farahani RMZ. Dental Treatment Duration as an Indicator of the Behavior of 3- to 9-Year-old Pediatric Patients in Clinical Dental Settings. J Contemp Dent Pract [Internet]. 2009 Sept; 10(5). Available from: http://www.thejcdp.com/journal/view/dentaltreatment-duration-as-an-indicator-of-thebehaviorof-3-to-9-year-ol. 


\section{Introduction}

Behavior guidance is the cornerstone of successful pediatric treatment. The factors influencing child behavior are either intrinsic (patient-centered) or extrinsic and dictate the behavior guidance strategies used by clinicians. A child's chronological age and cognitive level, temperament and personality characteristics, anxiety and fear, reaction to strangers, and previous dental experiences are examples of intrinsic factors. ${ }^{1}$ The extrinsic factors, e.g., maternal dental anxiety ${ }^{2-4}$ and parenting style ${ }^{5.6}$ exert their effects indirectly via modulation of intrinsic factors. Among these factors, the importance of treatment duration as an extrinsic determinant of child behavior has simply been overlooked.

Behaviors tend to deteriorate in a time-dependant manner. Longer dental visits are usually interpreted by pediatric patients as a sign of significant dental problems. Wright and Alpern demonstrated children who suspect a dental problem tend to exhibit more negative behavior. ${ }^{7}$ Finn suggested an almost inverse relationship between the cooperation of the patient and the length of time spent in the dental chair. ${ }^{.}$On the other hand, the effective accomplishment of several dental procedures in a short appointment is practically impossible. Decreasing the time of a dental visit at the expense of increased therapeutic sessions may be undesirable. Therefore, maintaining a balance between the treatment duration and efficient behavior management is of vital importance in pediatric dentistry. However, there is not a general consensus over the issue of optimal appointment length in pediatric dentistry.

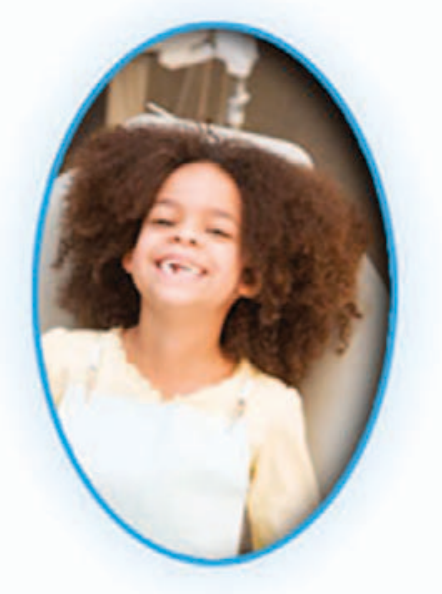

Shorter appointment times have long been advocated as a cooperation-enhancing approach in dealing with children in the dental setting. Finn states appointments for children should be limited to one-half hour in duration. ${ }^{-8}$ McDonald advocates dental appointments should be short until the patient gains self-confidence and rapport is established with the dentist. ${ }^{9}$ Conversely, there is support for longer treatment periods. Lenchner's study demonstrated longer appointments are not necessarily associated with a decline in behavior. ${ }^{10}$ Taylor et al. ${ }^{11}$ found the length of appointment is not significantly related to the child's behavior in the dental setting.

The existing controversy regarding the optimal duration of appointment may partially reflects the potential flaws in study design. The investigation of age groups rather than individual ages may lead to the blurring of the findings. The sustained attention span of a child patient is the major determinant of their reaction to treatment length. This progressively develops until the late school ages and hence necessitates a more meticulous evaluation of the correlation of chronological age and an apprehension-free treatment duration. Moreover, some confounding factors, e.g., pain, make the interpretation of the findings more difficult. The hypothesis of the present study is the length of appointment may affect the pediatric patients' behavior in a time-dependant and agedependant manner. Therefore, the aim of the study was to conduct a psychological evaluation of the association of patient's behavior with treatment duration at various chronological ages.

\section{Methods and Materials}

\section{Study Population}

A total of 450 children aged 3 to 9 years were included in the present study. The study was performed between February, 2007 and April, 2008. The subjects presented to the Department of Pediatric Dentistry of Tabriz University of Medical Sciences in Tabriz, East Azerbaijan, Iran, for the treatment of carious primary teeth. The selected subjects were in complete physical and mental health without any confounding medical history. Stratified random sampling was used for the selection of the subjects in any age group. The study population was allocated into six age groups: 
- Group A: Three to four years old $(\geq 3$ age $<4)$

- Group B: Four to five years old $(\geq 4$ age $<5)$

- Group C: Five to six years old $(\geq 5$ age $<6)$

- Group D: Six to seven years old $(\geq 6$ age $<7)$

- Group E: Seven to eight years old $(\geq 7$ age $<8)$

- Group F: Eight to nine years old $(\geq 8$ age $<9)$

The following criteria were considered for inclusion into the study:

- No history of dental treatment

- No history of post-traumatic stress disorders or specific phobia related to dental settings

- No history of unpleasant experiences in medical settings

- No previous experience of intraoral injections

- No history of pain secondary to pulpitis favoring a biased context due to especial pain syndromes such as hyperalgesia or allodynia

The study procedure as well as probable risks and discomforts were explained to the parents or legal guardians of the patients. The study design was approved by the research and ethics committees of Tabriz University of Medical Sciences.

\section{Study Procedure}

A pre-appointment visit was arranged during which behavior shaping of the study subjects was performed. Treatment visits consisted of a Type 1 preventive resin restoration (PRR), prophylaxis, and fluoride therapy. The total treatment duration was divided into three observational periods with an estimated time of 20 minutes for each procedure. The length of appointment was defined as the amount of time between entering and leaving the treatment area. The treatment regimen started with PRR, followed by the prophylaxis, and then completed with a fluoride application. Treatment selection was based on the patient's needs, absence of the need for local anesthesia, and the simplicity of treatment.

One pediatric dentist served as operator. The Type 1 PRR technique was used when pit and fissure decay was minimal. A very small round bur (No. $1 / 4$ or $1 / 2$ ) was used for caries removal. The preparation was confined to enamel. After selective enameloplasty, isolation by cotton rolls was achieved. The tooth was etched and a sealant was applied and cured (Phase 1). A dental prophylaxis was performed for cleaning and polishing the teeth as part of professionally administered fluoride therapy. All subjects received

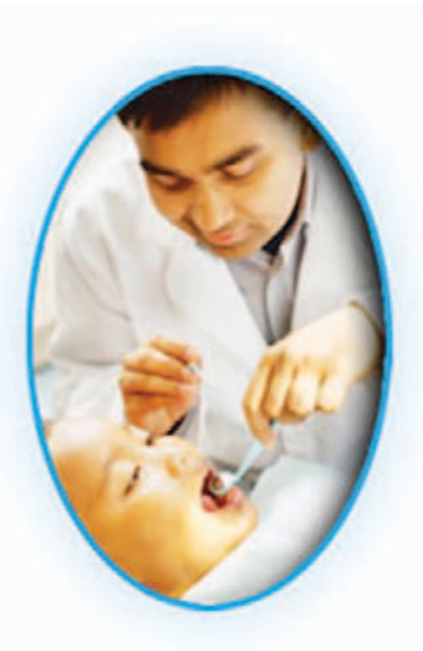

professional plaque removal (dental prophylaxis) using a rubber cup and low speed handpiece with a medium-grit prophylaxis paste (Prophy Paste with Fluoride, Oral-B, Belmont, CA, USA) followed by site-specific flossing (Phase 2). All patients received a single four-minute application of $1.23 \%$ APF fluoride solution for each quadrant (Phase 3). Parents were present during all phases of treatment which were completed in any situation regardless of whether a child was cooperative or not.

\section{Assessment of Children's Behavior}

A second dentist and one trained nurse assessed the children's behavior at the end of each treatment period using the sound, eye, and motor (SEM) scale. ${ }^{12}$ The assessment criteria of the SEM scale are based on three types of data, namely child sounds, eye signs, and body movements. The patients were observed through the entire procedure. On a random basis, a third investigator performed the SEM evaluation along with the second investigator to allow for the assessment of inter-examiner agreement of data.

\section{Statistical Analysis}

SPSS 14.0 analytical software (SPSS, Inc., Chicago, IL, USA) was used for data analysis. All quantitative data are presented as mean \pm standard deviation. Cronbach's alpha was used to determine the internal consistency reliability of the sound, eye, and motor modules of SEM scale. A repeated measures analysis of variance (ANOVA) was conducted to assess the withingroup differences between the average SEM scores of three temporal periods in any of the 
age groups. A mixed ANOVA was conducted to assess the main effects of age and time and their interaction. Post-hoc analysis of data was performed based on Scheffe's test. Polynomial and repeated contrasts were checked. Intraexaminer agreement of data was evaluated using paired-samples t-test. In the present study, $P<0.01$ was considered to indicate statistical significance.

\section{Results}

A total of 450 children aged 3 to 9 years were included in the present study. The intraexaminer agreement of SEM evaluation was 0.89 based on the correlation of test-retest values $(P<0.001)$. The test-retest difference of mean SEM values (0.25) was not statistically significant $(P=0.104)$.

Cronbach's alpha for constituent items of SEM scale was 0.775 demonstrating a high internal consistency reliability of sound, eye, and motor modules. The results of monovariate analysis of sound, eye, motor, and SEM values are presented in Table 1.

\section{Age-specific Within-group Analysis of SEM}

A repeated measures ANOVA was conducted to assess whether there were differences between the average SEM scores of three temporal periods in any of the age groups.

- Group A (3-4 year-old Age Group): Results indicated the participants in Group A reacted differently across the three periods, $\mathrm{F}(1.56$, 37.45) $=83.76, P<0.001$, eta $^{2}=0.777$. The means and standard deviations for agespecific SEM values are presented in Table 1. Examination of these means suggest the subjects' behavior deteriorated as treatment duration elongated. As depicted in Figure 1, the difference in mean SEM values between first and second 20 minutes was more than the difference between second and third periods.

- Group B (4-5 year-old Age Group): The behavior of children across three temporal periods differed significantly, $F(1.81,43.32)=$

Table 1. Mean (SD) of sound, eye, and motor modules and SEM score of evaluated age groups in three phases of treatment.

\begin{tabular}{|c|c|c|c|c|c|}
\hline Age Group & Phase & Sound & Eye & Motor & SEM \\
\hline \multirow{3}{*}{$\begin{array}{l}\text { Group A } \\
\text { (3-4 Year-olds) }\end{array}$} & $\mathrm{P} 1$ & $1.52(.77)$ & $1.60(.76)$ & $1.48(.65)$ & $4.60(1.35)$ \\
\hline & $\mathrm{P} 2$ & $2.80(.71)$ & $2.68(.69)$ & $2.20(.65)$ & $7.68(1.31)$ \\
\hline & P3 & $3.44(.65)$ & $3.00(.87)$ & $2.76(.93)$ & $9.20(1.22)$ \\
\hline \multirow{3}{*}{$\begin{array}{l}\text { Group B } \\
\text { (4-5 Year-olds) }\end{array}$} & $\mathrm{P} 1$ & $1.52(.51)$ & $1.44(.51)$ & $1.32(.48)$ & $4.28(.70)$ \\
\hline & $\mathrm{P} 2$ & $2.56(.51)$ & $2.36(.64)$ & $2.36(.57)$ & $7.28(.74)$ \\
\hline & $\mathrm{P} 3$ & $2.92(.71)$ & $2.84(.69)$ & $3.08(.86)$ & $8.84(1.46)$ \\
\hline \multirow{3}{*}{$\begin{array}{l}\text { Group C } \\
\text { (5-6 Year-olds) }\end{array}$} & $\mathrm{P} 1$ & $1.28(.46)$ & $1.40(.50)$ & $1.24(.44)$ & $3.92(1.04)$ \\
\hline & $\mathrm{P} 2$ & $1.96(.61)$ & $2.00(.71)$ & $1.36(.49)$ & $5.32(.45)$ \\
\hline & P3 & $1.88(.60)$ & $2.08(.64)$ & $2.16(.69)$ & $6.12(1.33)$ \\
\hline \multirow{3}{*}{$\begin{array}{l}\text { Group D } \\
\text { (6-7 Year-olds) }\end{array}$} & P1 & $1.32(.48)$ & $1.44(.51)$ & $1.28(.46)$ & $4.04(.98)$ \\
\hline & P2 & $1.36(.49)$ & $1.44(.51)$ & $1.32(.48)$ & $4.12(.97)$ \\
\hline & P3 & $1.64(.44)$ & $1.64(.49)$ & $1.72(.54)$ & $5.00(1.00)$ \\
\hline \multirow{3}{*}{$\begin{array}{l}\text { Group E } \\
\text { (7-8 Year-olds) }\end{array}$} & $\mathrm{P} 1$ & $1.20(.41)$ & $1.36(.40)$ & $1.28(.46)$ & $3.84(.75)$ \\
\hline & P2 & $1.20(.41)$ & $1.24(.44)$ & $1.24(.44)$ & $3.68(.75)$ \\
\hline & P3 & $1.48(.51)$ & $1.56(.51)$ & $1.40(.50)$ & $4.44(.77)$ \\
\hline \multirow{3}{*}{$\begin{array}{l}\text { Group F } \\
\text { (8-9 Year-olds) }\end{array}$} & $\mathrm{P} 1$ & $1.12(.33)$ & $1.24(.44)$ & $1.16(.37)$ & $3.52(.59)$ \\
\hline & $\mathrm{P} 2$ & $1.12(.33)$ & $1.16(.37)$ & $1.20(.41)$ & $3.48(.65)$ \\
\hline & P3 & $1.20(.41)$ & $1.36(.49)$ & $1.24(.44)$ & $3.80(.76)$ \\
\hline
\end{tabular}




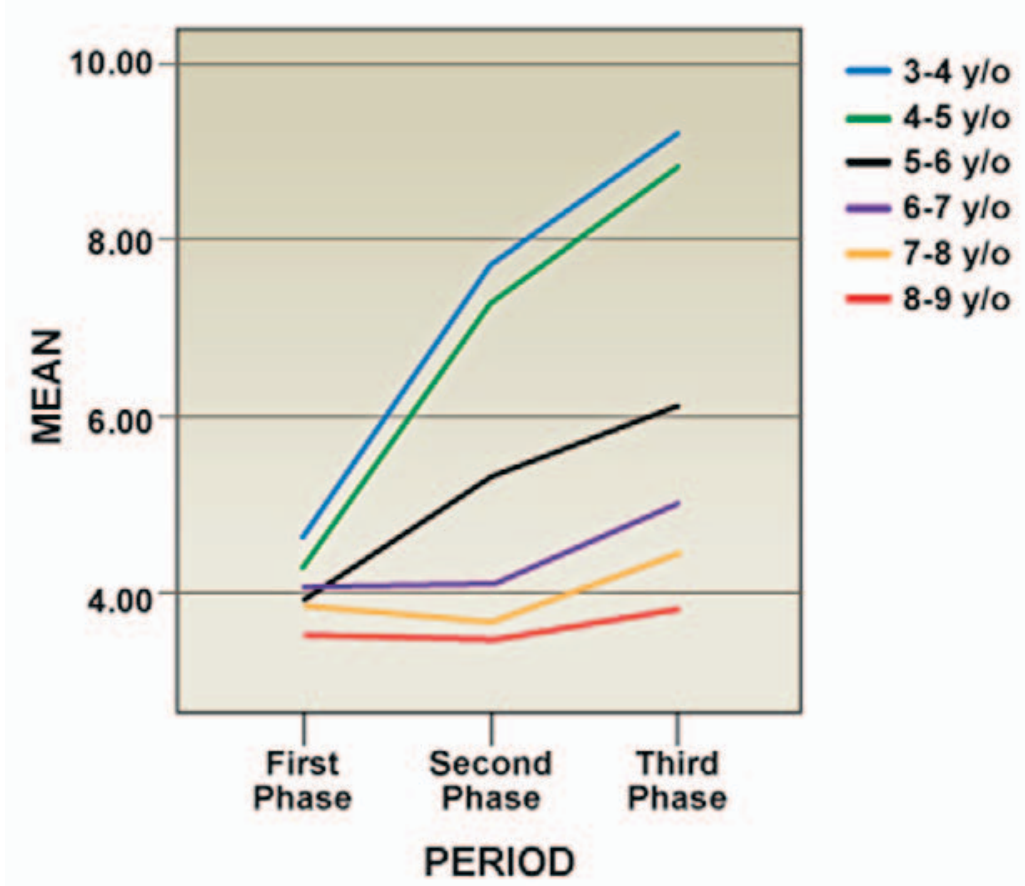

Figure 1. Diagrammatic representation of mean SEM scores for various age groups.

83.76, $\mathrm{P}<0.001$, eta $^{2}=0.85$. The inspection of mean SEM values (Table 1) revealed the children's behavior deteriorated in a timedependant manner.

- Group C (5-6 year-old Age Group): The within-group difference of three periods was statistically significant in this group, $F$ $(2,48)=23.50, P<0.001$, eta $^{2}=0.495$. As evidenced by mean SEM values, a similar trend toward misbehavior parallel with the increased duration of treatment was observed. The repeated contrasts revealed the SEM difference between the second and the third phases of therapy was not significant, $\mathrm{F}(1$, 24) $=4.92, P=0.036$, eta $^{2}=0.17$.

- Group D (6-7 year-old Age Group): The participants in Group D reacted differently across the three periods, $F(2,48)=6.66$, $P=0.003$, eta ${ }^{2}=0.217$. The mean SEM difference between phase one and two was not statistically significant, $F(1,24)=0.074$, $P=0.79$, eta ${ }^{2}=0.003$. However, the mean SEM values between the second and the third phases differed significantly, $F(1,24)=9.66$, $P=0.004$, eta $^{2}=0.293$.

- Group E (7-8 year-old Age Group): The behavior of children across three temporal periods differed significantly, $\mathrm{F}(1.88$, 45.03) $=83.76, P=0.002$, eta $^{2}=0.246$. The mean SEM difference between the first and the second phases was not statistically significant, $F(1,24)=1.000, P=0.327$, eta $^{2}$ $=0.04$. However, the mean SEM values between the second and the third phases differed significantly, $F(1,24)=12.134, P=$ 0.002, eta $^{2}=0.246$.

- Group F (8-9 year-old Age Group): The within-group SEM difference of three periods was not statistically significant in this group, $\mathrm{F}$ $(1.87,44.83)=1.41, \mathrm{P}=0.254$, eta $^{2}=0.056$. The time-dependant behavior of SEM was neither of linear $(F(1,24)=2.042, P=0.166$, eta $\left.^{2}=0.078\right)$ nor of quadratic $(F(1,24)=$ $0.907, P=0.350$, eta $^{2}=0.036$ ) nature.

\section{Between-group Analysis of SEM}

A mixed ANOVA was conducted to assess whether there were differences between the average SEM scores considering the main effects and interaction of time and chronological age.

The results indicated a significant effect of treatment duration, $\mathrm{F}(1.69,255.01)=188.29, \mathrm{P}$ $<0.001$, and of chronological age, $\mathrm{F}(\mathrm{I}, 144)=$ $115.82, P<0.001$. Moreover, the primary effects of time and chronological age was qualified by a significant interaction between time and chronological age, $\mathrm{F}(8.86,255.01)=115.82$, $P<0.001$. Although behavior deteriorates as 
the duration of the treatment is extended this indicates the trend in each age group shows specific characteristics. Post-hoc analysis of the data was performed based using Scheffe's test. The results of post-hoc analysis of data are presented in Table 2.

\section{Regression Analysis}

Hierarchical multiple regression was conducted to determine the best linear combination of chronological age and treatment duration for predicting negative behavior of children according to the SEM score. The means, standard deviations, and intercorrelations can be found in Table 3.
The chronological age significantly predicted children's misbehavior, $\mathrm{F}(1,448)=313.04, \mathrm{P}<$ 0.001 . The adjusted $R$ squared value was 0.41 . This indicates $41 \%$ of the variance in SEM score was explained by the model used in the study. However, the model, with the addition of the treatment duration, more efficiently predicted the SEM score, $F(2,447)=339.30, P<0.001$. The superiority of Model 2 (age + time) compared to Model 1 (age) was evidenced by the increase in the adjusted $R^{2}$ value from $R^{2}=0.41$ to an $R^{2}=0.60$, $F(1,447)=215.60, P<0.001$. The beta weights, presented in Table 4, suggest chronological age contributes most to predicting children's behavior and treatment duration also contributes to this prediction.

Table 2. $P$ values of post-hoc between-group analysis of SEM scores.

\begin{tabular}{|l|c|c|c|c|c|c|}
\hline $\begin{array}{c}\text { Age } \\
\text { Group }\end{array}$ & $\mathbf{3 - 4}$ yo* $^{*}$ & $\mathbf{4 - 5}$ yo & $\mathbf{5 - 6}$ yo & $\mathbf{6 - 7}$ yo & $\mathbf{7 - 8}$ yo & $\mathbf{8 - 9}$ yo \\
\hline 3-4 yo & 0.008 & $<0.001$ & $<0.001$ & $<0.001$ & $<0.001$ & $<0.001$ \\
\hline $4-5$ yo & - & - & $<0.001$ & $<0.001$ & $<0.001$ & $<0.001$ \\
\hline 5-6 yo & - & - & - & 0.002 & $<0.001$ & $<0.001$ \\
\hline $6-7$ yo & - & - & - & - & 0.326 & 0.001 \\
\hline 7-8 yo & - & - & - & - & - & 0.365 \\
\hline 8-9 yo & - & - & - & - & - & - \\
\hline *yo: years old. & & & & & \\
\hline
\end{tabular}

Table 3. Intercorrelation of age and treatment duration variables with SEM score.

\begin{tabular}{|l|c|c|}
\hline \multicolumn{1}{|c|}{ Variable } & \multicolumn{2}{c|}{ Intercorrelation } \\
\cline { 2 - 3 } & Age & Treatment Duration \\
\hline SEM score & $-0.64^{*}$ & $0.44^{*}$ \\
\hline Age & - & $0.00^{* *}$ \\
\hline Treatment Duration & - & - \\
\hline${ }^{*} \mathrm{P}<0.001,{ }^{* *} \mathrm{P}=0.5$ & & \\
\hline
\end{tabular}

Table 4. Hierarchical multiple regression analysis for chronological age and treatment duration.

\begin{tabular}{|l|c|c|c|}
\hline \multicolumn{1}{|c|}{ Variable } & B & Standard error of B & Beta \\
\hline Age & -0.77 & 0.036 & -0.64 \\
\hline Treatment duration & 1.10 & 0.075 & 0.44 \\
\hline Constant & 5.67 & 0.205 & - \\
\hline $\mathrm{R}^{2}=0.60, \mathrm{~F}(2,447)=339.30,<0.001$ & \multicolumn{3}{|l|}{} \\
\hline
\end{tabular}


Table 5. ROC curve analysis of predictive ability of age and treatment duration for critical SEM score of five.

\begin{tabular}{|c|c|c|c|}
\hline Variable & $\begin{array}{l}\text { SEM over five if } \\
\text { variable > or }=\text { to: }\end{array}$ & Sensitivity & 1-Specificity** \\
\hline \multirow{6}{*}{ Age } & 3 & 0.936 & 0.632 \\
\hline & 4 & 0.856 & 0.296 \\
\hline & 5 & 0.705 & 0.099 \\
\hline & 6 & 0.497 & 0.013 \\
\hline & 7 & 0.252 & 0.000 \\
\hline & 8 & 0.00 & 0.000 \\
\hline \multirow{3}{*}{ Treatment duration } & $20 \mathrm{~min}$ & 0.00 & 0.921 \\
\hline & $40 \mathrm{~min}$ & 0.245 & 0.507 \\
\hline & $60 \mathrm{~min}$ & 0.537 & 0.921 \\
\hline \multicolumn{4}{|c|}{$\begin{array}{l}\text { *Probability of SEM } \leq 5 \text { when the value of the variable is below those mentioned. } \\
{ }^{* *} \text { The probability that a variable has its value below those mentioned when SEM } \leq 5 \text {. }\end{array}$} \\
\hline
\end{tabular}

\section{Discussion}

The aim of the present study was psychological evaluation of the association of patient's behavior with treatment duration at various chronological ages. The findings suggest treatment duration affects the pediatric patient's behavior through age-dependant conduct.

There is a lack of general consensus on optimal duration of treatment in pediatric dentistry. The review of the traditional literature suggests treatment should be limited to 15 minutes for children up to five years of age. ${ }^{10,11}$ Treatment duration should be shorter than an average appointment while allowing for a specific procedure to be completed because short treatment duration gives the child a feeling of having cooperated with the clinical team. The findings of the present study are in accordance with these suggestions. Children up to five years of age were found to be more likely to demonstrate negative behavior as treatment time is extended. For example, when 4-year-old pediatric patients were subjected to treatment durations as long as 40 minutes, the risk of exhibiting a negative behavior was almost inevitable (85\% [age] + 24\% [time] $>100 \%)$ as shown in Table 5. It may be advisable to limit the treatment duration to 20 minutes for children up to six years of age.
On the contrary, Lenchner's study ${ }^{10}$ demonstrated longer appointments are not necessarily associated with a decline in behavior. Moreover, Taylor et al. ${ }^{11}$ found the length of appointment is not significantly related to children's behavior in dental settings. The difference in the study design may partially reflect the observed disagreement. Lenchner's study had a relatively small sample size and categorized the subjects based on age groups rather than individual ages. Moreover, an important flaw of the aforementioned study was the assessment of the frequencies of observed behaviors as a dichotomous variable, which may lead to elimination or masking of certain important details.

The emergence of misbehavior exhibits certain characteristics in any age group which may be of clinical significance. In 3-5 year-old children there is a rapid deterioration of behavior between 20 and 40 minutes during a treatment procedure. Thereafter, the aggravation of behavior is much slower as evidenced by the significant quadratic trend (Figure 1). It is not advisable to extend the treatment duration beyond 20 minutes for this age group. The emergence of misbehavior in 5 year-old children is more gradual and rather linear and therefore easier to control in clinical settings. Therefore, through application of proper behavior guidance strategies, the treatment duration may be extended to 40 minutes and 
even further. For children aged 6-9 years of age, the treatment duration may be extended up to 60 minutes parallel with the use of behavior guidance strategies.

It may be desirable to address the mechanisms through which treatment duration affects the behavior of pediatric patients. Two different sets of psychological attributes mediate the scenario. Some of these factors are transient. Anxiety and fear are examples of transient mediators of a child's behavior in a dental operatory. On the other hand, motor skills and attention span of children are intrinsic psychological attributes of children which remain unaffected by situational environmental stimuli. These psychological attributes exert their effect via influencing various levels of behavior.

Development of attentional networks is necessary for maintenance of a mutual communication between the dentist and the patient. Age-related differences are well documented in the behavioral developmental literature. ${ }^{13-15}$ Evidence has been obtained demonstrating the developmental component of sustained attention is generally linear between the ages of 2.5 and 4.5 years. $.16,17$ Some evidence of a quadratic trend in focused attention between the ages of 1 and 3.5 years has also been obtained. $\frac{16}{}$ The development of sustained attention reaches a plateau or "levels off" around the age of 5 which persists until the age of 6 has been demonstrated. $\frac{18}{{ }^{18}}$ Therefore, the development of sustained attention may partially explain the observed differences among age groups. Moreover, the statistically-defined critical age of 5 (ROC curve analysis in Figure 2 and Table 5) is highly concordant with the plateau in development of sustained attention. The ROC (receiver operating characteristic) curve shows specificity and sensitivity of the tested variables, and here, explains the differences in behavior, indicated by the SEM score, according to different age groups and various treatment durations, with a cut-off point of 5 set for the SEM score.

While treatment duration is not a triggering agent for anxiety behavior, it may aggravate it. Interestingly, treatment duration of anxious patients is on average $40 \%$ more than nonanxious patients going through the same operation has been demonstrated..$^{19}$ Therefore, observed positive feedback leads to a defective cycle in which anxiety extends the treatment duration and vice versa.

There are several limitations inherent in the present study design. The most important limitations are related to the minimization of confounding factors in an attempt to enhance

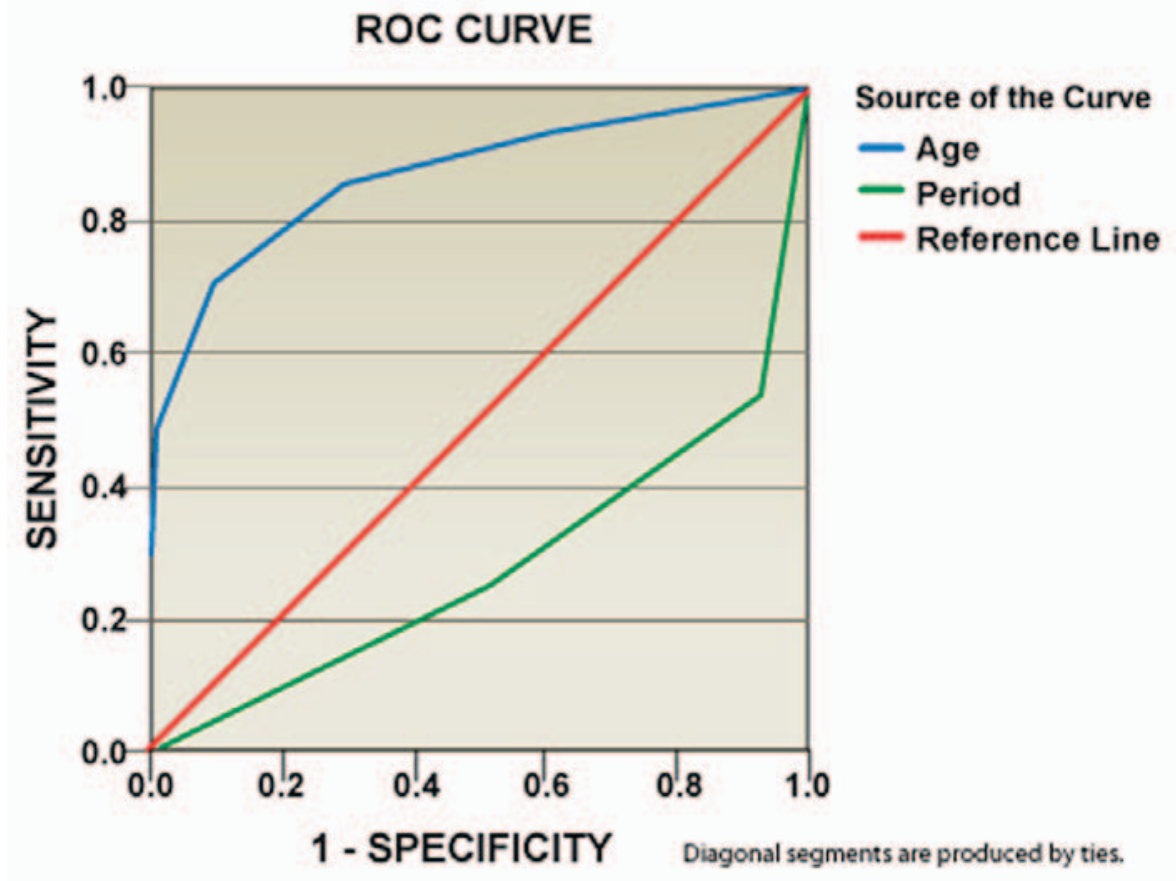

Figure 2. ROC curves for predictive ability of age and treatment duration for critical SEM score of five. 
the internal validity of the study at the expense of external validity. The study sample consisted of a relatively homogeneous population of cooperative children without a previous dental experience (Frankl's Class III or IV). However, the real clinical situation involves a relatively heterogeneous population. While this homogeneity enhances the validity of the present findings, the extrapolation of these findings should be carefully weighed considering each clinical scenario.

\section{Conclusion}

Within the limitation of this study, treatment duration may affect the behavior of pediatric patients in combination with chronological age and should be considered in the formulation of an efficient treatment plan. Thus, it may be helpful to present conclusions as general guidelines:

- For children aged 3-5 years, the treatment duration should be strictly limited to 20 minutes.

- For 5-year-old children, the upper limit of optimal treatment duration is 20 minutes. However, it may be extended up to 40 minutes.

- For 6-year-old children the upper limit of optimal treatment duration is 40 minutes. However, an extension of 20 minutes (overall duration of 60 minutes) may be acceptable.

- For children aged 7-9 years, the upper limit of optimal treatment duration is 60 minutes.

\section{Clinical Significance}

The findings of this study suggest appropriate pediatric behavior management should include thoughtful scheduling of appointments according to a treatment plan formulated with consideration of the effects of age and appointment length.

\section{References}

1. American Academy of Pediatric Dentistry Clinical Affairs Committee--Behavior Management Subcomittee; American Academy of Pediatric Dentistry Council on Clinical Affairs--Committee on Behavior Guidance. Guideline on behavior guidance for the pediatric dental patient. Pediatr Dent. 2005-2006; 27 Suppl 2:92-100.
2. Klingberg G, Berggen U, Carlsson SG, Noren JG. Child dental fear: Cause related factors and clinical effects. Eur J Oral Sci. 1995; 103:405-412.

3. Lee CY, Chang YY, Huang ST. The clinically related predictors of dental fear in Taiwanese children. Int J Paediatr Dent. 2008; 18:415-22.

4. Peretz B, Nazarian Y, Bimstein E. Dental anxiety in a students pediatric dental clinic: Children, parents and students. Int J Paediatr Dent. 2004; 14:192-198.

5. Weiner AA, Forgione A, Weiner LK, Hwang J. Potential fear-provoking patient experiences during treatment. Gen Dent. 2000; 48:466-71.

6. Aminabadi NA, Farahani RM. Correlation of parenting style and pediatric behavior guidance strategies in the dental setting: preliminary findings. Acta Odontol Scand. 2008; 66:99-104.

7. Wright GZ, Alpern MD. Variables influencing children's cooperative behavior at the first dental visit. ASDC J Dent Child. 1971; 38:124-8.

8. Finn SB. Child management in the dental office. In: Finn SB, editor. Clinical Pedodontics. Philadelphia: WB Saunders; 1998. p. 39.

9. McDonald RE, Avery DR, Dean JA. Dentistry for the Child and Adolescent. St. Louis: Mosby. 8th ed. 2004. p 47.

10. Lenchner V. The effect of appointment length on behavior of the pedodontic patient and his attitude toward dentistry. J Dent Child. 1966; 33:61-74

11. Taylor MH, Moyer IN, Peterson DS. Effect of appointment time, age, and gender on children's behavior in a dental setting. ASDC J Dent Child. 1983; 50:106-10.

12. Wright GZ, Weinberger SJ, Marti R, Plotzke $O$. The effectiveness of infiltration anesthesia in the mandibular primary molar region.

Pediatr Dent. 1991; 13:278-83.

13. Ridderinkhof KR, van der Stelt O. Attention and selection in the growing child: views derived from developmental psychophysiology. Biol Psychol. 2000; 54:55-106.

14. Rueda MR, Fan J, McCandliss BD, Halparin JD, Gruber DB, Lercari LP, Posner MI. Development of attentional networks in childhood. Neuropsychologia. 2004; 42: 1029-40.

15. Posner MI, Sheese BE, Odludas Y, Tang Y. Analyzing and shaping human attentional networks. Neural Netw. 2006; 19:1422-9. 
16. Ruff AH, Lawson KR. Development of sustained, focused attention in young children during free play. Dev Psychol. 1990; 26: 85-93.

17. Ruff AH, Lawson KR, Parrinello R, Weissberg R. Long-term stability of individual differences in sustained attention in the early years. Child Dev. 1990; 61:60-75.

18. Sarid M, Breznitz Z. Developmental aspects of sustained attention among 2 to 6 -year-old children. Int J Behav Dev. 1997; 21:303-312.

19. Filewich RJ, Jackson E, Shore H. Effects of dental fear on efficiency of dental procedures. J Dent Res. 1981; 60:Abstract \#895.

\section{About the Authors}

\section{Naser Asl Aminabadi, DDS, MSc} (Corresponding Author)

Dr. Aminabadi is an Associate Professor in the Department of Pedodontics of the School of Dentistry at Tabriz University of Medical Sciences in Tabriz, East Azerbaijan, Iran. His research interests include behavioral management of children, orthodontic correction of dentoskeletal anomalies, and root canal therapy of primary teeth.

e-mail: aslaminabadi@gmail.com

\section{Sina Ghertasi Oskouei, DDS}

Dr. Ghertasi is a Research Assistant in the Department of Pedodontics of the School of Dentistry at Tabriz University of Medical Sciences in Tabriz, East Azerbaijan, Iran. His research interests are in the areas of basic and behavioral sciences related to dental medicine.

e-mail: ghertasis@gmail.com

\section{Ramin Mostofi Zadeh Farahani, DDS}

Dr. Farahani is a Research Assistant in the Institute of Dental Research of Westmead Millennium Institute, University of Sydney, Sydney, NSW, Australia. His primary research interests include wound healing and tissue regeneration in addition to his interest in the field of pediatric dentistry.

e-mail: r.mostofi@gmail.com 